\title{
Title: Pay-it-forward to improve influenza vaccine uptake and public engagement among children and older adults in China
}

Dan Wu ${ }^{1,2,3, *, \#}$, Chenqi Jin ${ }^{2,4, \#}$, Khaoula Bessame ${ }^{1}$, Fanny Fong-Yi Tang ${ }^{1,5}$, Jason J. Ong ${ }^{1,6,7}$, Zaisheng Wang ${ }^{2,8}$, Yewei Xie ${ }^{2,9}$, Mark Jit ${ }^{10}$, Heidi J. Larson ${ }^{10}$, Tracey Chantler ${ }^{11}$, Leesa Lin $^{10,12}$, Wenfeng Gong ${ }^{13}$, Fan Yang ${ }^{14}$, Fengshi Jing ${ }^{2,15}$, Shufang $\mathrm{Wei}^{2}$, Weibin Cheng ${ }^{15}$, Yi Zhou $^{16,17}$, Nina Ren ${ }^{15}$, Shuhao Qiu ${ }^{18}$, Jianmin Bao ${ }^{19}$, Liufen Wen ${ }^{20}$, Qinlu Yang ${ }^{21}$, Junzhang $\operatorname{Tian}^{15}$, Weiming Tang ${ }^{2,4,22, *,}$, Joseph D. Tucker ${ }^{1,2,22, \S}$

1. Department of Clinical Research, Faculty of Infectious and Tropical Diseases, London School of Hygiene and Tropical Medicine, London, UK.

2. University of North Carolina at Chapel Hill Project-China, Guangzhou, China

3. West China School of Public Health, Sichuan University, Chengdu, China

4. Dermatology Hospital of South Medical University, Guangzhou, China

5. Li Ka Shing Faculty of Medicine, The University of Hong Kong, Hong Kong, China

6. Central Clinical School, Faculty of Medicine, Monash University, Melbourne, Australia

7. Melbourne Sexual Health Centre, Alfred Health, Melbourne, Australia

8. School of Health and Related Research, The University of Sheffield, Sheffield, UK.

9. Duke Global Health Institute, Duke University, North Carolina, U.S.

10. Faculty of Epidemiology and Population Health, London School of Hygiene and Tropical Medicine, London, UK.

11. Faculty of Public Health and Policy, London School of Hygiene and Tropical Medicine, London, UK.

12. Laboratory of Data Discovery for Health (D24H), Hong Kong Science Park, Hong Kong, China

13. Beijing Representative Office, Bill \& Melinda Gates Foundation, Beijing, China

14. Institute of Population Research, Peking University, Beijing, China

15. Guangdong Secondary Provincial General Hospital, Guangzhou, China

16. Zhuhai Center for Disease Control and Prevention, Zhuhai, China

17. Faculty of Medicine, Macau University of Science and Technology, Macau SAR, China

18. Vaccination Clinic, Yangshan Health Centre, Qingyuan, China.

19. Fenghuang Community Health Service Centre, Zengcheng, China.

20. Xinghua Community Health Service Centre, Guangzhou, China. 
21. Community representative, Guangzhou, China.

22. Institute for Global Health and Infectious Diseases, School of Medicine, University of North Carolina at Chapel Hill, North Carolina, USA

\footnotetext{
${ }^{\#}$ Denotes co-first authors

${ }^{\S}$ Denotes co-senior authors

* Corresponding author
}

Dr Dan Wu, Department of Clinical Research, London School of Hygiene and Tropical Medicine, Keppel St, London WC1E 7HT, United Kingdom, dan.wu@1shtm.ac.uk

\author{
*Alternative corresponding author \\ Dr Weiming Tang, 2. University of North Carolina at Chapel Hill Project-China, Guangzhou, \\ China, weiming_tang@med.unc.edu
}

\begin{abstract}
Background: China has low seasonal influenza vaccination rates among children and older adults. This quasi-experimental pragmatic trial examined the effectiveness of a pay-it-forward intervention on influenza vaccine uptake among children and older adults compared to standard of care (user-paid vaccination) and free vaccination strategies in China. Pay-itforward is a community-engaged social innovation in which people receive a free influenza vaccination from a local group and are then asked if they would like to donate financially and/or create a postcard message to promote vaccination among future individuals.

Methods: We iteratively co-created the pay-it-forward intervention with the community through public engagement and a participatory hackathon. We implemented the three study arms in three clinics (rural, suburban, urban) in Guangdong Province, China. A total of 225 children aged between six months and eight years old and 225 older adults (60 years old or above) were recruited into the study. Regression methods were used to compare influenza
\end{abstract}


vaccine uptake (administrative records) and vaccine confidence (self-report) between the three arms.

Results: Among all participants, 55/150 (36.7\%) in the standard of care arm received an influenza vaccine. 111/150 (74.0\%) of people in the pay-it-forward arm received an influenza vaccine. $114 / 150(76.0 \%)$ of people offered free vaccination received an influenza vaccination. Similar trends were observed among children and older adults. The pay-itforward arm had significantly higher vaccine confidence when compared to the standard of care arm. In the pay-it-forward arm, 107/111 (96.4\%) of participants donated money for subsequent vaccinations and 19 of 60 invited (31.7\%) created postcard messages.

Conclusions: Pay-it-forward was effective in improving influenza vaccine uptake and engagement among children and older adults. Our data have implications for pro-social interventions to enhance influenza uptake in the many countries where influenza vaccines are available for a fee.

Trial registration: ChiCTR2000040048

\section{Introduction}

In mainland China, an average of 10 people die from influenza-related illnesses each hour. ${ }^{1}$ Influenza vaccination is the most effective way to prevent morbidity and mortality attributable to influenza. ${ }^{2}$ Influenza vaccine is increasingly important during COVID-19 because it might help reduce risks of acquiring SARS-CoV-2 that causes COVID-19. ${ }^{3,4}$ The Chinese Center for Diseases Control and Prevention (China CDC) guidelines recommend influenza vaccination for high-risk populations, including children aged below 5 years old and older adults (people older than 60 years old). However, influenza immunization policies widely vary, ${ }^{5}$ and most cities in China do not provide free influenza vaccines to high-risk individuals. A meta-analysis reported less than one-fifth of children and older adults in China 
received an influenza vaccine in the past year. ${ }^{6}$

There are several reasons for low influenza vaccination uptake in China. ${ }^{6,7}$ First, most people in China are unaware of influenza vaccination and many people are hesitant about vaccine safety and effectiveness. ${ }^{8}$ Second, there is minimal public engagement in vaccinations. ${ }^{9}$ Despite a strong rationale for public engagement, few programs engage the public regarding influenza vaccinations. Third, there are limited public resources to support influenza vaccination among high-risk populations. The influenza vaccine is largely not covered by mandatory health insurance schemes and, as a result, most people have to pay \$8.5-23.5 outof-pocket to be vaccinated. ${ }^{10}$ Innovative strategies are needed to improve influenza vaccine uptake.

Pay-it-forward is a community-engaged social innovation, which has an individual receive a free influenza vaccine and a hand-written postcard message co-created by previous participants informing them that someone else has paid for them to receive a free vaccine. ${ }^{11}$ After they receive vaccination, they are asked if they would like to support the vaccination of a subsequent person (supplementary figures: Fig 1). Our previous pay-it-forward studies focused on increasing testing for sexually transmitted infections among sexual minorities. The pay-it-forward arm had a chlamydia and gonorrhea dual test uptake of 56\% compared to $18 \%$ in the standard of care arm, where participants had to pay out-of-pocket. ${ }^{12}{ }^{13}$ In addition, over $90 \%$ of participants donated to the rolling finance pool, ${ }^{12,13}$ and qualitative data showed that trust in health services improved among participants in the pay-it-forward arm. ${ }^{14}$

In this quasi-experimental pragmatic trial, we assessed the effectiveness of a pay-it-forward intervention to increase influenza vaccination uptake at three sites among children (aged between 6 months and 8 years) and older adults (aged 60 or above) in comparison to free vaccination and the current standard of care in China.

\section{Methods}


Guangdong is a subtropical province in southern China with a population of over 120 million. In southern China, influenza is prevalent throughout the year. ${ }^{15}$ In this study, we selected three research sites where influenza vaccination was only available on a for-fee basis. These three study sites were: a rural site (Yangshan County, Qingyuan City), a suburban site (Zengcheng District, Guangzhou City), and an urban site (Tianhe District, Guangzhou, City). Study sites included community health centers (primary care facilities providing day-to-day healthcare in China) and vaccine centers. Clinics were selected because they had sufficient influenza vaccines in stock and health professionals (nurses, doctors) familiar with influenza vaccination.

This study consisted of three stages 1) co-creation of the intervention with stakeholders and engagement strategies during a three-day hackathon; 2) a feasibility pilot to inform the recruitment process and sample size calculations; and 3) a pay-it-forward quasi-experimental pragmatic trial to evaluate the effectiveness of the intervention.

\section{Co-creation of intervention}

Our team of three individuals joined a participatory hackathon from November 4-6 2019, to co-create the pay-it-forward intervention. Co-creation is an iterative, bidirectional partnership between researchers and the public to develop new ideas. ${ }^{16}$ Participants included potential end users, public health practitioners, health innovators, communication experts and vaccine experts. We mapped out the following elements of the study: 1) key stakeholders of the study; 2) potential user journeys; 3) engagement strategies; 4) behavioral mechanisms; and 5) donation strategies. Community engagement strategies used in this study included the following: inviting community members to design postcards (Supplementary figures: Fig 2); working in partnership with a local older adult to develop a video to explain pay-it-forward; inviting study participants to create hand-written postcard messages during recruitment for future participants (Supplementary figures: Fig 3); and engaging local community staff in implementing the quasi-experimental study, including having one-to-two community staff members at each study site to help adjust recruitment and communication efforts to the local dialect. 
Pilot

Before the quasi-experimental study, we carried out a feasibility pilot at the rural study site from January to April 2020, which occurred during the social distancing period due to COVID-19 in China. The primary outcome of the study was influenza vaccination uptake. The purpose of the pilot was to finalize the pay-it-forward intervention process, assess feasibility, and estimate effect size to inform power calculations. This pilot demonstrated that, in the pay-it-forward arm, 90.9\% (40/44) of participants received an influenza vaccine and 93\% (37/40) of participants donated funds. Thirteen of 57 participants $(22.3 \%)$ in the standard of care arm received a vaccine.

\section{Sample size calculation}

We stratified sample size calculations by age groups, given the differences between children and older adults. Based on our pilot data, we anticipated that the proportion of vaccine uptake in the standard-of-care arm was $30 \%$, and the proportion of vaccine uptake in the pay-itforward arm was $80 \%$, a significance level of 0.025 ; therefore, a sample size 100 (50 in the control arm and 50 in the intervention) would give us $90 \%$ power to test the proportion difference with a margin of $10 \%$. We increased the sample size by $50 \%$ to allow for secondary analyses, resulting in a sample size of 75 for each age group in each arm. In addition, we included a free vaccine arm with the same sample size as the other two arms. This free vaccination arm was included because it has important implications for policy and global relevance to countries that already provide free influenza vaccinations. In sum, we required the enrollment of 225 children and 225 older adults in order to have sufficient power.

\section{Quasi-experimental pragmatic trial}

This trial evaluated the pay-it-forward intervention arm against both the standard of care and a free vaccination program implemented in rural, suburban, and urban study sites. Each study site implemented all study arms and recruited participants were chronologically allocated (non-random) into the specified study arms because of practical considerations. Influenza vaccine services are usually available in China from September to April. Influenza vaccine availability is idiosyncratic at specific health facilities because of the periodical supply and 
procurement system in local settings. We allocated study arms to ensure a stable supply of vaccines (Supplementary figures: Fig 4: time-based recruitment). At each site, the standard of care arm was followed by the pay-it-forward arm. Despite discussions with health authorities and vaccine manufacturers, study sites encountered lapses in supply. The duration of time needed to recruit each study arm was related to the availability of vaccine and the number of people willing to participate.

The inclusion criteria for this study differed by age group and were determined according to China's national influenza vaccine guidelines. ${ }^{17}$ Childhood eligibility criteria included the following: aged between six months and eight years old; no acute moderate or severe illnesses; eligible to receive an influenza vaccine based on clinical evaluation from a physician; has a legal guardian (e.g. a parent or grandparent) who lives in China and consents to participate in the study; and has not received an influenza vaccine in the past year. Older adult eligibility criteria included the following: $\geq 60$ years old; no acute moderate or severe illness; eligible to receive an influenza vaccine based on clinical evaluation from a physician; capable of making informed decisions and consenting to participate in the study; and have not received an influenza vaccine in the past year. If multiple people in a family were eligible to join the study, we only allowed one person to join. All eligible children and older adults presenting to these sites were invited to participate by local medical staff involved in the study during the recruitment periods.

Ethical approval for this study was obtained from the institutional review boards at the London School of Hygiene \& Tropical Medicine (approval number 19100) and the Zhuhai Center for Disease Control. Online consent was obtained from guardians of children and older adults.

\section{Procedures}

Among all potential participants visiting the selected clinics, health care workers assessed eligibility for the study based on inclusion criteria and introduced eligible participants to project staff.

Standard of care Participants recruited in the standard of care arm were provided with a 
brief introduction to the influenza vaccine by project staff using a pamphlet about influenza and influenza vaccination (Supplementary figures: Fig 5). They were then asked if they were willing to pay out of pocket at the standard market price (US\$8.5-23.5 depending on the market price of vaccines provided at the clinic) to receive an influenza vaccination. Those who agreed to pay were screened for vaccination eligibility, and those without any contraindications received the vaccine.

Pay-it-forward Participants recruited in the pay-it-forward arm were provided with the same introductory pamphlet about influenza and influenza vaccination. Project staff then explained the pay-it-forward program, including its purpose, the opportunity to receive one dose of influenza vaccination for free, and the opportunity to donate money towards someone else's vaccine dose and write postcard messages (Supplementary figures: Fig 6). Participants were told that the normal price to receive an influenza vaccine, including administration fees were RMB 56(US\$ 8.5) for children and 153(US\$ 23.5) for adults, and that previous participants had donated money to cover the costs and had also created handwritten postcards for them.

If the participants decided to receive vaccination, they were asked prior to receiving the vaccination whether they were willing to donate any amount of money into a pool of funds to support subsequent participants in receiving the same vaccine. They were assured that the donation was entirely voluntary, and any donation amount was acceptable and would not affect whether they received a vaccination or subsequent care. They were also invited to write anonymous postcard messages for future participants. A donation collection box was provided on-site for those who preferred to donate cash. A QR code using WeChat (a multifunctional social mobile app embedded with anonymous money transfer functions) was provided to those who chose to make online donations.

Donations were used to support the vaccination of subsequent participants and aggregated data on donation amounts were made publicly available on the website and WeChat newsletter of Social Entrepreneurship to Spur Health (a research hub in the UNICEF/UNDP/World Bank/WHO Special Programme for Research and Training in Tropical Diseases Social Innovation in Health Initiative). COVID-19 conditions at the rural site prevented participants from creating handwritten postcards during some periods of the 
trial.

Free vaccination Participants in the free vaccination arm were invited to participate using the same introductory pamphlet and were provided with free influenza vaccination. They did not receive any community-created messages about the pay-it-forward program.

Participation in each arm was voluntary and anonymous. After introducing the intervention, all participants were asked to complete a short, self-administered online questionnaire to collect information about sociodemographic characteristics and attitudes towards influenza vaccines. Vaccine confidence in importance, safety, and effectiveness were measured using survey items adapted to assess influenza vaccine confidence in China. ${ }^{18,19}$ Those who had difficulty reading the questionnaire were assisted by the project and healthcare staff on-site. A small gift worth around RMB10 (US\$1.5) was given to each participant after completing the questionnaire survey.

\section{Data collection}

Data collection was conducted from September 2020 to March 2021. The study collected the following information: administrative data recorded by research staff using a standard information tracking sheet including the number of invited and participating individuals, the number of participants who received the vaccine, the number of individuals who donated and the amount donated in the pay-it-forward arm, as well as survey data through a selfadministered survey instrument (supplementary questionnaire). Administrative and survey data were linked using numerical IDs. We collected information about the number of participants in the pay-it-forward arm who donated and corresponding donation amount, and those who created a postcard text for subsequent people. Costs associated with each arm were collected for an economic evaluation.

\section{Data analysis}

Descriptive analyses were conducted to summarize each arm's sociodemographic and behavioral characteristics, participation rate, and vaccination rate. We used a Chi-squared test to investigate differences in vaccination uptake between the standard of care, pay-it-forward, 
and free vaccination arms. We ran multivariable logistic regression to examine the association between vaccine uptake and interventions after adjusting for age, sex, study sites, education, occupation, income and marital status. We also summarized the participants' donations in the pay-it-forward arm, and compared proportions of participants between rural, suburban and urban sites who contributed US\$7.6 (close to a child vaccine cost) or more. All data were analyzed using SPSS Version 25 and SAS.

\section{Cost Analysis}

A decision tree was built to calculate and compare the costs and outcomes of the three influenza vaccination arms examined in the quasi-experimental study. We evaluated the costs of all three arms using a micro-costing approach and reported this in 2020 USD. The costs of implementing each strategy were estimated using invoices, onsite staff's self-reporting the wages of healthcare workers, and estimated opportunity costs of community staff's time (supplementary economic evaluation). The analysis was performed from the perspective of the healthcare provider, the Guangdong Department of Health. The time horizon considered was the duration of the seasonal influenza vaccination program. We reported the total economic and financial cost for each arm, the cost per person vaccinated, and the incremental cost-effectiveness ratios. Incremental economic costs were greater for PIF compared to free vaccination because of additional costs related to volunteer time in the PIF design, as well as recruitment and donation process associated with the start-up, and recurrent costs. However, incremental financial costs were greater for free vaccination compared to pay-it-forward because financial costs were obtained from subtracting donation contributions from the total economic cost.

\section{Results}

In total, 254 children's caregivers and 262 older adults were approached at the three study sites (Fig 1). Forty-six people declined to participate, and 30 had recently received the influenza vaccine. In total, 450 participants were enrolled and completed the survey, including 150 in the pay-it-forward arm, 150 in the free vaccine arm, and 150 in the standard of care arm. All 450 responses were screened for completeness and included in the final 
statistical analyses.

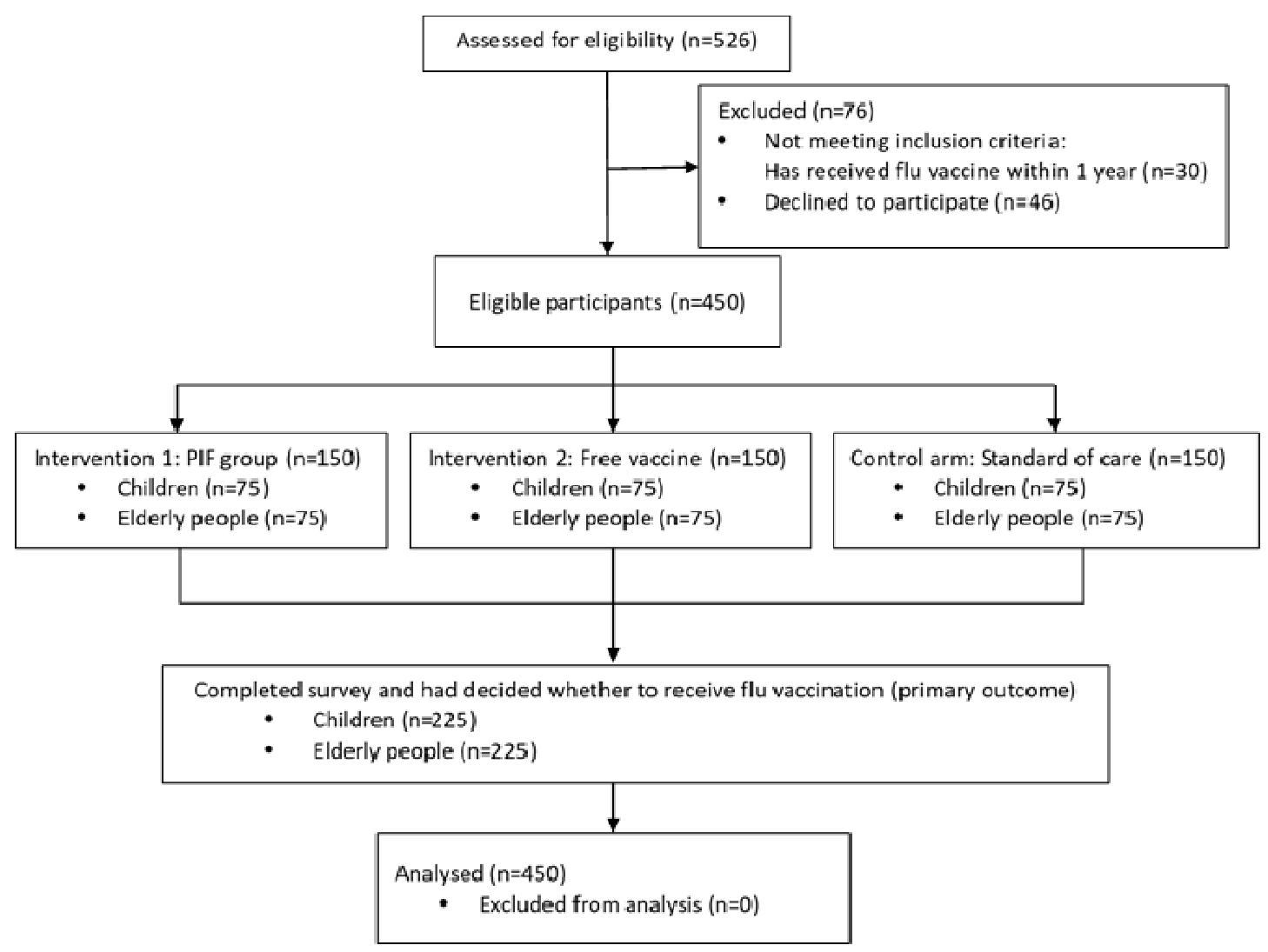

\section{Fig 1 Quasi-experimental study flow chart}

Characteristics of caregivers and older adult participants were similar across the three arms (Table 1). Overall, 111 (74.0\%) of 150 pay-it-forward participants, $114(76.0 \%)$ of 150 participants offered free vaccination, and 55 (36.7\%) of 150 participants in the standard of care arm received influenza vaccination ( $\chi^{2}$ test $p<0.001$; fig 2 , supplementary tables: table 1$)$. Among children, the pay-it-forward arm had an uptake rate of $88.0 \%(66 / 75)$ compared to $53.3 \%(40 / 75)$ in the standard of care arm. Among older adults, the pay-it-forward arm had an uptake rate of $60.0 \%(45 / 75)$ compared to $20.0 \%$ (15/75) in the standard of care arm. Differences in uptake between the pay-it-forward and standard of care arms remained statistically significant for both groups after adjusting for study site and educational level (Supplementary tables: table 1). Uptake in the pay-it-forward and free vaccination arms were 
not significantly different for both age groups.

Table 3 suggests that people in the pay-it-forward arm were more likely to receive the vaccine compared to people in the standard of care arm (adjusted odd ratio $(\mathrm{aOR})=7.9$, 95\% CI [3.2-19.7] among children; aOR=6.1, [2.8-13.2] among older adults). Free-of-charge vaccination participants had greater odds than those in the standard of care arms to receive a vaccination $(\mathrm{aOR}=5.5$, [2.4-12.6] among children; $\mathrm{aOR}=9.1$, [4.2-20.0] among older adults). People in the pay-it-forward arm had higher vaccine confidence in safety compared to people in the standard of care arm $(\mathrm{aOR}=2.25$, [1.27- 4.01], with greater odds than those in the free vaccination arm $(\mathrm{aOR}=1.55,[0.91-2.63])$ (Table 3$)$. Similar trends were observed in confidence in vaccine effectiveness and importance.

Study participants contributed to the development of influenza vaccination materials in several important ways. 19/60 (31.7\%) people created handwritten postcards for subsequent participants. Six postcard designs were subsequently used to explain the pay-it-forward system to potential participants. Most handwritten messages expressed general good wishes. In addition, we co-created a video and one local older adult contributed to this video design, implementation, and evaluation (supplementary video link).

Among 111 participants in the pay-it-forward arm who received the influenza vaccine, 107 (96.4\%) donated money, with a total contribution of US\$597.62. Donations covered $36.0 \%$ of vaccination costs in the pay-it-forward arm. The median donation was US\$4.6. Only $30 \%$ of donors in the rural site contributed US $\$ 7.6$ or more compared to $61.9 \%$ in the suburban and $40.0 \%$ in urban sites (supplementary Fig 7 and supplementary table 2).

The total financial cost of implementing an influenza vaccination intervention for children and older adults was US\$2,725 for the standard of care arm, US $\$ 4,477$ for the pay-it-forward arm, and US\$4,665 for the free vaccination arm. The incremental cost for each treatment arm, the incremental number of people vaccinated, and the incremental cost-effectiveness ratios (ICERs) based on financial and economic costs are shown in Table 4 and in the supplementary economic evaluation file. Based on the ICER obtained from comparing pay-itforward to the standard of care, the financial cost required per additional person vaccinated 
medRxiv preprint doi: https://doi.org/10.1101/2021.10.30.21265713; this version posted November 1, 2021. The copyright holder for this preprint

(which was not certified by peer review) is the author/funder, who has granted medRxiv a license to display the preprint in perpetuity.

It is made available under a CC-BY-NC-ND 4.0 International license .

was US\$31.29. The financial cost required per additional person vaccinated was US\$62.67

in the free vaccination arm.

When economic costs are considered, the economic cost of implementing an influenza

vaccination intervention for children and older adults was US\$3,557 for standard of care,

US $\$ 5,062$ for pay-it-forward, US $\$ 4,665$ for the free vaccination arm. 
Table 1 Sample characteristics of recruited child caregivers and older adults in Guangdong Province, China, 2021 (N=450)

\begin{tabular}{|c|c|c|c|c|c|c|c|c|}
\hline & \multicolumn{4}{|c|}{ Child caregiver group } & \multicolumn{4}{|c|}{ The older adult group } \\
\hline & $\begin{array}{l}\text { Standard of } \\
\text { care } \\
(\mathrm{N}=75)\end{array}$ & $\begin{array}{l}\text { Pay-it-forward } \\
(\mathrm{N}=75)\end{array}$ & $\begin{array}{l}\text { Free } \\
\text { vaccination } \\
(\mathrm{N}=75)\end{array}$ & $\mathrm{p}$ value & $\begin{array}{l}\text { Standard of } \\
\text { care } \\
(\mathrm{N}=75)\end{array}$ & $\begin{array}{l}\text { Pay-it-forward } \\
(\mathrm{N}=75)\end{array}$ & $\begin{array}{l}\text { Free } \\
\text { vaccination } \\
(\mathrm{N}=75)\end{array}$ & $\mathrm{p}$ value \\
\hline Age & $35.91(10.3)$ & $36.71(9.7)$ & $36.95(8.9)$ & 0.357 & $69.53(6.4)$ & $66.52(6.7)$ & $68.59(6.0)$ & 0.988 \\
\hline Sex & & & & 0.975 & & & & 0.489 \\
\hline Men & $17(22.7)$ & $16(21.3)$ & $17(22.7)$ & & $20(26.7)$ & $27(36.0)$ & $23(30.7)$ & \\
\hline Women & $58(77.3)$ & $59(78.7)$ & $58(77.3)$ & & $55(73.3)$ & $48(64.0)$ & $52(69.3)$ & \\
\hline Education & & & & 0.005 & & & & 0.459 \\
\hline Elementary school & $8(10.7)$ & $4(5.3)$ & $4(5.3)$ & & $33(44.0)$ & $26(34.7)$ & $29(38.7)$ & \\
\hline Middle school & $45(60.0)$ & $26(34.7)$ & $35(46.7)$ & & $31(41.3)$ & $42(56.0)$ & $35(46.7)$ & \\
\hline Undergraduate or above & $22(29.3)$ & $45(60.0)$ & $36(48.0)$ & & $11(14.7)$ & $7(9.3)$ & $11(14.7)$ & \\
\hline $\begin{array}{l}\text { Occupation } \\
\text { Unemployed }\end{array}$ & $20(26.7)$ & $21(28.0)$ & $22(29.3)$ & 0.281 & $53(70.7)$ & $58(77.3)$ & $60(80.0)$ & 0.785 \\
\hline Peasant & $1(1.3)$ & $1(1.3)$ & $5(6.7)$ & & $19(25.3)$ & $15(20.0)$ & $13(17.3)$ & \\
\hline Employed & $54(72.0)$ & $53(70.7)$ & $48(64.0)$ & & $3(4.0)$ & $2(2.7)$ & $2(2.7)$ & \\
\hline Annual income (USD) & & & & 0.963 & & & & 0.664 \\
\hline $0-1860$ & $19(25.3)$ & $23(30.7)$ & $20(26.7)$ & & $38(50.7)$ & $28(37.3)$ & $28(37.3)$ & \\
\hline $1860-9300$ & $22(29.3)$ & $24(32.0)$ & $21(28.0)$ & & $29(38.7)$ & $36(48.0)$ & $37(49.3)$ & \\
\hline $9300-1,8600$ & $20(26.7)$ & $16(21.3)$ & $19(25.3)$ & & $7(9.3)$ & $10(13.3)$ & $8(10.7)$ & \\
\hline$>1,8600$ & $14(18.7)$ & $12(16.0)$ & $15(20.0)$ & & $1(1.3)$ & $1(1.3)$ & $2(2.7)$ & \\
\hline Marital status & & & & 0.658 & & & & 0.149 \\
\hline $\begin{array}{l}\text { Single, divorced, } \\
\text { separated or widowed }\end{array}$ & $4(5.3)$ & $4(5.3)$ & $2(2.7)$ & & $20(26.7)$ & $20(26.7)$ & $11(14.7)$ & \\
\hline $\begin{array}{l}\text { Married or living with a } \\
\text { partner }\end{array}$ & $71(94.7)$ & $71(94.7)$ & $73(97.3)$ & & $55(73.3)$ & $55(73.3)$ & $64(85.3)$ & \\
\hline
\end{tabular}


Table 2: Multivariable logistic regression to compare vaccine uptake rates of three arms by age groups in Guangdong Province, China, 2020-2021 (N=450)

\begin{tabular}{lllll}
\hline \multirow{2}{*}{ Arms/Age groups } & \multicolumn{2}{l}{ Children $(\mathrm{N}=225)$} & \multicolumn{2}{l}{ Older adults $(\mathrm{N}=225)$} \\
& COR $(95 \% \mathrm{CI})$ & AOR $(95 \% \mathrm{CI})$ & COR $(95 \% \mathrm{CI})$ & AOR (95\% CI) \\
\hline Standard of care & Ref & Ref & Ref & Ref \\
Pay-it-forward & $6.42(2.80,14.73)$ & $7.93(3.20,19.68)$ & $6.00(2.89,12.46)$ & $6.07(2.81,13.15)$ \\
& $\mathrm{p}<0.0001$ & $\mathrm{p}<0.0001$ & $\mathrm{p}<0.0001$ & $9.104(4.15,19.96)$ \\
Free vaccine & $5.09(2.324,11.153)$ & $5.48(2.38,12.63)$ & $8.00(2.81,16.81)$ & $\mathrm{p}<0.0001$ \\
\hline
\end{tabular}

AOR: Model adjusted for age, sex, study sites, education level, occupation, income, and marital status. 
medRxiv preprint doi: https://doi.org/10.1101/2021.10.30.21265713; this version posted November 1, 2021. The copyright holder for this preprint (which was not certified by peer review) is the author/funder, who has granted medRxiv a license to display the preprint in perpetuity.

It is made available under a CC-BY-NC-ND 4.0 International license .

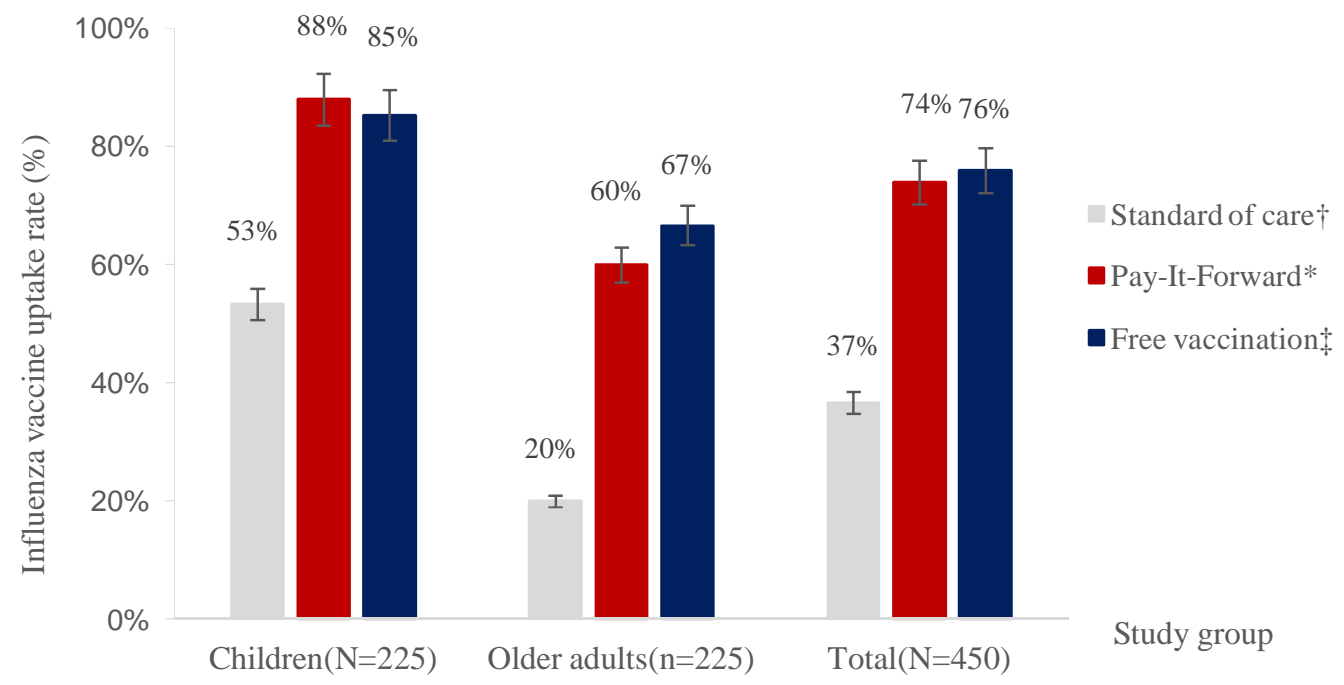

$\dagger$ Standard of care refers to self-paid vaccination, *Pay-it-forward refers to the community based intervention that offered a free vaccine and participants could chose to donate to support more people; $\ddagger$ Free vaccination arm in which participants received

Fig 2: Influenza vaccine uptake rates by intervention arms and age group in Guangdong Province, China, 2020-2021 (N=450) 
Table 3 Association between study arm and vaccine confidence in influenza vaccine in Guangdong Province, China, 2020-2021 (N=450)

\begin{tabular}{|c|c|c|c|c|c|c|c|c|c|}
\hline \multirow[t]{2}{*}{ Study arm } & \multicolumn{3}{|c|}{ Vaccine confidence - Safety } & \multicolumn{3}{|c|}{ Vaccine confidence - Importance } & \multicolumn{3}{|c|}{ Vaccine confidence - Effectiveness } \\
\hline & $\begin{array}{l}\mathrm{cOR}^{3} \\
(95 \% \mathrm{CI})^{6}\end{array}$ & $\begin{array}{l}\mathrm{aOR}^{4} \\
(95 \% \mathrm{CI})\end{array}$ & $\mathrm{P}$ value ${ }^{5}$ & $\begin{array}{l}\text { cOR } \\
(95 \% \mathrm{CI})\end{array}$ & $\begin{array}{l}\text { aOR } \\
(95 \% \mathrm{CI})\end{array}$ & $P$ value & $\begin{array}{l}\text { cOR } \\
(95 \% \mathrm{CI})\end{array}$ & $\begin{array}{l}\text { aOR } \\
(95 \% \mathrm{CI})\end{array}$ & $\mathrm{P}$ value \\
\hline Standard of care ${ }^{1}$ & Reference & Reference & 0.02 & Reference & Reference & 0.004 & Reference & Reference & 0.004 \\
\hline Free vaccine & $\begin{array}{l}1.59 \\
(0.95-1.67)\end{array}$ & $\begin{array}{l}1.55 \\
(0.91-2.63)\end{array}$ & & $\begin{array}{l}2.50 \\
(1.42-4.43)\end{array}$ & $\begin{array}{l}2.50 \\
(1.38-4.52)\end{array}$ & & $\begin{array}{l}1.71 \\
(1.04-2.82)\end{array}$ & $\begin{array}{l}1.70 \\
(1.01-2.85)\end{array}$ & \\
\hline Pay-it-forward ${ }^{2}$ & $\begin{array}{l}2.42 \\
(1.38-4.25)\end{array}$ & $\begin{array}{l}2.25 \\
(1.27-4.01)\end{array}$ & & $\begin{array}{l}3.39 \\
(1.82-6.29)\end{array}$ & $\begin{array}{l}3.17 \\
(1.66-6.05)\end{array}$ & & $\begin{array}{l}3.34 \\
(1.89-5.89)\end{array}$ & $\begin{array}{l}3.15 \\
(1.75-5.64)\end{array}$ & \\
\hline
\end{tabular}

Out-of-pocket payment for the influenza vaccine was the standard of care.

${ }^{2}$ In addition to free influenza vaccines, the pay-it-forward study arm received community engagement messages as well as the opportunity to make a donation to support the vaccination of other members of the community.

${ }^{3} \mathrm{cOR}=$ crude odds ratio

${ }^{4} \mathrm{aOR}=$ adjusted odds ratio

${ }^{5}$ P-value obtained using Likelihood Ratio Tests

${ }^{6} \mathrm{CI}=$ confidence interval

${ }^{7}$ Estimates were adjusted for age, gender, education level, income level. 
Questionnaire number :

Table 4: Economic evaluation of the influenza vaccine strategies

\begin{tabular}{|c|c|c|c|c|c|}
\hline $\begin{array}{l}\text { Treatment } \\
\text { group }\end{array}$ & $\begin{array}{l}\text { Financial } \\
\text { cost (USD) }\end{array}$ & $\begin{array}{l}\text { Incremental } \\
\text { cost (USD) }\end{array}$ & $\begin{array}{l}\text { Number of } \\
\text { people } \\
\text { vaccinated }\end{array}$ & $\begin{array}{l}\text { Incremental } \\
\text { number of } \\
\text { people } \\
\text { vaccinated }\end{array}$ & $\begin{array}{l}\text { ICER (USD } \\
\text { per person } \\
\text { vaccinated) }\end{array}$ \\
\hline $\begin{array}{l}\text { Standard-of- } \\
\text { care }\end{array}$ & 2725 & & 55 & & \\
\hline $\begin{array}{l}\text { Pay-it- } \\
\text { forward }\end{array}$ & 4477 & 1752 & 111 & 56 & 31.29 \\
\hline $\begin{array}{l}\text { Free } \\
\text { vaccination }\end{array}$ & 4665 & 188 & 114 & 3 & 62.67 \\
\hline $\begin{array}{l}\text { Treatment } \\
\text { group }\end{array}$ & $\begin{array}{l}\text { Economic } \\
\text { cost (USD) }\end{array}$ & $\begin{array}{l}\text { Incremental } \\
\text { cost (USD) }\end{array}$ & $\begin{array}{l}\text { Number of } \\
\text { people } \\
\text { vaccinated }\end{array}$ & $\begin{array}{l}\text { Incremental } \\
\text { number of } \\
\text { people } \\
\text { vaccinated }\end{array}$ & $\begin{array}{l}\text { ICER (USD } \\
\text { per person } \\
\text { vaccinated) }\end{array}$ \\
\hline $\begin{array}{l}\text { Standard-of- } \\
\text { care }\end{array}$ & 3557 & & 55 & & \\
\hline $\begin{array}{l}\text { Free } \\
\text { vaccination }\end{array}$ & 4665 & 1109 & 114 & 59 & 18.79 \\
\hline $\begin{array}{l}\text { Pay-it- } \\
\text { forward }\end{array}$ & 5062 & 397 & 111 & -3 & Dominated \\
\hline
\end{tabular}

$\boldsymbol{I C E R}=$ incremental cost-effectiveness ratio; $\boldsymbol{U S D}=$ United States dollars (2020)

\section{Discussion}

Low influenza vaccination rates among high-risk populations are a major health problem in low and middle-income countries. Our quasi-experimental study assessed the effectiveness of a pay-it-forward influenza vaccination intervention to improve uptake and engagement. Our study contributes to the literature by determining the effectiveness of a social innovation using a quasi-experimental study, developing new methods for influenza vaccination public engagement, and identifying a new method to enhance influenza vaccine uptake. Our data suggest that the pay-it-forward strategy may increase influenza vaccine uptake among high-risk individuals compared to the current self-pay strategy for vaccination. This strategy substantially increased vaccine uptake compared to the standard of care, elicited financial contributions, improved vaccine confidence, and co-created participatory messages.

We found that children and older adults who took part in pay-it-forward had higher influenza vaccine uptake than they did if they needed to self-pay for vaccination. This 
finding is consistent with previous intervention studies using pay-it-forward to improve health services uptake. ${ }^{12,13}$ The effect of pay-it-forward might be related to the reduced costs associated with vaccination, enhanced public engagement, or both. The pay-it-forward arm had a similar vaccination rate to that of the free vaccine arm. This suggests that asking participants to make some financial contribution to support the vaccination of subsequent participants did not deter them from participating.

We also observed that, among those enrolled in the pay-it-forward arm, nearly all voluntarily donated to support another person in receiving an influenza vaccine, including those with a low annual income from a study site in a poor rural area. Compared to standard of care, the pay-it-forward arm had a higher financial cost, but increased the number of people vaccinated. The incremental financial cost per person vaccinated was lower than the median cost (US \$50.78) per additional enrollee vaccinated from a systematic review published in $2018 .{ }^{20}$ Donations collected using a pay-it-forward system can support more individuals in receiving influenza vaccine services and can potentially reduce the financial burden for local governments. Pay-itforward could also potentially transition from out-of-pocket payments to governmentfunded influenza vaccine programs.

Pay-it-forward has additional social benefits; it generated many messages aimed at driving influenza vaccine uptake. This is a rare example of public engagement in an influenza vaccination program. ${ }^{9}$ Public engagement is central to the success of public health programs; given that some engagement methods (in-person events) could facilitate influenza transmission, ${ }^{21}$ it is especially important to identify public engagement methods that are safe and effective. Engaging the community in vaccination services through cultivating kindness and reciprocity may also strengthen community solidarity, and increase confidence in vaccine services. ${ }^{14,22}$

The study has several limitations. First, although our study was implemented after COVID-19 lockdowns were lifted, all sites were heavily focused on COVID-19 prevention, COVID-19 vaccination, and related COVID-19 activities. This caused some delays in recruitment despite the availability of influenza vaccines. At the same 
time, this demonstrates the feasibility of pay-it-forward, even during an event as disruptive as COVID-19. Second, we examined people from only three sites. However, our sites all had a high influenza prevalence, included different settings (rural, suburban, urban), and reflected common pathways for vaccination in China. Third, our study did not capture granular data on implementation. Future effectiveness research to examine different pay-it-forward implementation strategies is needed to differentiate effective components and determine optimal pay-it-forward practices. Finally, the study was mainly implemented by our project staff with assistance from local health workers. It remains unclear how feasible it is to decentralize implementation and integrate pay-it-forward into existing vaccine services.

Our study has implications for research, implementation, and policy. From a research perspective, this study expands the limited literature on public engagement in influenza vaccine programs. It demonstrates how social innovation can engage key communities in the implementation process and build confidence in influenza vaccination. This might help address vaccine hesitancy and anti-vaccine movements. The success of the pay-it-forward initiative and different donation levels across three study sites shows the potential to mobilize financial resources between areas with different economic status (e.g., mobilize financial resources from economically betteroff areas to subsidize essential preventive services for people in more impoverished areas). Randomized controlled trials and qualitative research are needed to better understand the implementation of this system and integrate this intervention within health systems.

Pay-it-forward may be particularly relevant in the large number of countries that charge fees for influenza vaccines, which contributes to disparities in influenza vaccine uptake. Developing pay-it-forward programs could help financially support expanded influenza vaccination programs in these settings while also generating community-engaged messages. Further pay-it-forward influenza vaccination research could help explore how to expand vaccine programs in a more equitable and prosocial manner. 


\section{Funding support}

This work received financial support from Bill \& Melinda Gates Foundation (OPP1217240), and the National Institute for Health Research (NIHR).

\section{Acknowledgement}

We appreciate support from Dr. David Sarley from Bill \& Melinda Gates Foundation for his contribution and support to the early development of the intervention package. We are grateful for comments from Dr. Jing Li at West China School of Public Health Sichuan University to help improve clarity of the paper. We thank Xu Chen, Xiaolin Qiu, Yuxin Ni, Yuan Xiong, Ruoyu Zhu, Jie Fan, Yumeng Du from University of North Carolina at Chapel Hill Project-China, Kaiyi Han from Faculty of Public Health and Policy, London School of Hygiene and Tropical Medicine for their help with data collection, video production, data visualization and interpretation.

\section{References}

1. Li, L., et al. Influenza-associated excess respiratory mortality in China, 2010-15: a population-based study. The Lancet Public Health 4, e473-e481 (2019).

2. Mameli, C., Cocchi, I., Fumagalli, M. \& Zuccotti, G. Influenza vaccination: effectiveness, indications, and limits in the pediatric population. Frontiers in pediatrics 7, 317 (2019).

3. Conlon, A., Ashur, C., Washer, L., Eagle, K.A. \& Bowman, M.A.H. Impact of the influenza vaccine on COVID-19 infection rates and severity. American journal of infection control (2021).

4. Candelli, M., et al. Effect of influenza vaccine on COVID-19 mortality: a retrospective study. Internal and Emergency Medicine, 1-7 (2021).

5. Principi, N., Camilloni, B., Esposito, S. \& Group, E.V.S. Influenza immunization policies: Which could be the main reasons for differences among countries? Human vaccines \& immunotherapeutics 14, 684-692 (2018).

6. Wang, Q., et al. Influenza vaccination coverage of population and the factors influencing influenza vaccination in mainland China: A meta-analysis. Vaccine 36, 7262-7269 (2018).

7. Wang, J., et al. Low awareness of influenza vaccination among pregnant women and their obstetricians: a population-based survey in Beijing, China. Human vaccines \& immunotherapeutics 15, 2637-2643 (2019).

8. Song, Y., et al. Increasing seasonal influenza vaccination among high risk groups in China: Do community healthcare workers have a role to play? Vaccine 35, 4060-4063 (2017).

9. Thomas, R.E. \& Lorenzetti, D.L. Interventions to increase influenza vaccination rates of those 60 years and older in the community. The Cochrane database of systematic reviews $\mathbf{5}$,

CD005188-CD005188 (2018). 
10. NA. How much does an influenza vaccine (trivalent and quadrivalent) cost?, Vol. 2021 (Wuhan.com, 2020).

11. Tang, W., et al. How kindness can be contagious in healthcare. Nature Medicine 27, 11421144 (2021).

12. Li, K.T., et al. Pay-it-forward strategy to enhance uptake of dual gonorrhea and chlamydia testing among men who have sex with men in China: a pragmatic, quasi-experimental study. The Lancet. Infectious diseases 19, 76-82 (2019).

13. Yang, F., et al. Pay-it-Forward Gonorrhea and Chlamydia Testing Among Men Who Have Sex With Men in China: A Randomized Controlled Trial. The Lancet. Infectious diseases (2020).

14. Li, K.T., et al. A Secondary Mixed Methods Analysis of a Pay-it-Forward Gonorrhea/Chlamydia Testing Program Among Men Who Have Sex With Men in China. Sexually transmitted diseases 47, 395-401 (2020).

15. Shu, Y.-L., et al. Dual seasonal patterns for influenza, China. Emerging infectious diseases 16, 725-726 (2010).

16. Finley, N., Swartz, T.H., Cao, K. \& Tucker, J.D. How to make your research jump off the page: Co-creation to broaden public engagement in medical research. PLOS Medicine 17, e1003246 (2020).

17. China CDC. Technical guideline for influenza vaccination in China Vol. 2019 (China CDC, Beijing, 2018).

18. Wei, Z., Sun, X., Yang, Y., Zhan, S. \& Fu, C. Seasonal influenza vaccine hesitancy profiles and determinants among Chinese children's guardians and the elderly. Expert Review of Vaccines, 1-10 (2021).

19. de Figueiredo, A., Simas, C., Karafillakis, E., Paterson, P. \& Larson, H.J. Mapping global trends in vaccine confidence and investigating barriers to vaccine uptake: a large-scale retrospective temporal modelling study. The Lancet 396, 898-908 (2020).

20. Anderson, L.J., et al. The Cost of Interventions to Increase Influenza Vaccination: A Systematic Review. American journal of preventive medicine 54, 299-315 (2018).

21. Shobugawa, Y., Fujiwara, T., Tashiro, A., Saito, R. \& Kondo, K. Social participation and risk of influenza infection in older adults: a cross-sectional study. BMJ open 8, e016876-e016876 (2018).

22. Konrath, S. \& Brown, S. The effects of giving on givers. in Health and social relationships: The good, the bad, and the complicated. 39-64 (American Psychological Association, Washington, DC, US, 2013). 